\title{
Frequency of Hypercholesterolemia in Recent Onset Type - 2 Diabetics with Microalbuminuria
}

\author{
Muhammad Adnan Hasham, ${ }^{1}$ Sakhawat Abbas, ${ }^{2}$ Mirza Ilyas Baig, ${ }^{3}$ Fawad Ahmad Randhawa ${ }^{4}$
}

\begin{abstract}
Amongst diabetes, type 2 diabetes encompasses more than $80 \%$ and even higher percentage is present in unindustrialized countries. Kidney disease in diabetic patients is clinically characterized by increasing rates of urinary albumin excretion, starting from no albuminuria, which progresses to microalbuminuria, macroalbuminuria and eventually to End Stage Renal Disease. Dyslipidemia is observed in patients with DM and microalbuminuria. Total cholesterol increases significantly with albuminuria.

Objectives: Frequency of hypercholesterolemia in recent onset type-2 diabetics with microalbuminuria.

Methodology: The study was conducted in Department of Endocrinology \& Metabolism, Services Hospital Lahore. Study was conducted and completed over a period of six months from $15^{\text {th }}$ September 2014 to $15^{\text {th }}$ March 2015. It was a cross-sectional survey with non-probability, purposive sampling technique. 200 patients fulfilling the criteria were enrolled. Informed
\end{abstract}

\footnotetext{
${ }^{1}$ Senior Registrar, Endocrinology Unit \& Diabetes Management Center, Services Hospital, Lahore

${ }^{2}$ Consultant Physician, Kot Khawaja Saeed Hospital Lahore

${ }^{3}$ Associate Professor, Fatima Memorial Hospital, Lahore

${ }^{4}$ Assistant Professor of Endocrinology

King Edward Medical University
}

Date of Submission: 14-12-2016

Date of Acceptance for Publication: 10-05-2017

Conflict of Interest: None

Funding Source: None

\section{Contribution}

All Authors have contributed in Study Design, Data Collection, Data Analysis, Data Interpretation, Manuscript Writing and Approval. consent was obtained. Demographic information of each patient was noted. Urine was obtained and samples were sent to the laboratory of the hospital and reports were assessed. If the value of albumin is raised in urine analysis (as per operational definition) then albuminuria was labeled. In patients who were diagnosed as with albuminuria, total cholesterol was noted. If cholesterol was $>240 \mathrm{mg} / \mathrm{dl}$, then hypercholesterolemia was labeled by researcher himself.

Conclusion: The results of the study conclude that frequency of microalbuminuria among patients of newly diagnosed type- 2 diabetic mellitus is in agreement with other studies while the frequency of hypercholesterolemia in patients with microalbuminuria was also recorded higher which needs special physicians attention for its timely management so that patients can be prevented from severe cardio vascular morbidities and mortality.

Keywords: Diabetes Mellitus Type 2, microalbuminuria, hypercholesterolemia.

\section{Introduction}

Diabetes mellitus (DM) is the metabolic syndrome that takes away a lot of economic resources for the developing world. ${ }^{1}$ Globally, as of 2010, an estimated 285 million people had diabetes, with type 2 making up about $90 \%$ of the cases. ${ }^{2}$ Diabetes Mellitus is on an alarming rise especially in the last two decades. ${ }^{1}$ According to a recent survey, this disease has affected around 240 million people worldwide. ${ }^{3}$ As far as disease burden in Pakistan is concerned, prevalence of newly diagnosed diabetes was $5.1 \%$ in men and $6.8 \%$ in women in urban areas. ${ }^{4}$

Diabetic nephropathy or microalbuminuria is the leading cause of renal failure. Microalbuminuria is defined as albumin excretion of $30-299 \mathrm{mg} / 24$ hours. 
Diabetic patients with microalbuminuria typically progress to proteinuria and overt diabetic nephropathy. According to one study, microalbuminuria is already present in around $7 \%$ of patients with type II diabetes at the time of diagnosis. ${ }^{5}$ In a study, the prevalence of microalbuminuria in diabetic patients was $24.2 \% .^{6}$ In a Chinese study, Among diabetic patients hypercholesterolemia was more frequent in patients compared with diabetic patients without microalbuminuria $(62.1 \%$ vs. $36.5 \%)^{7}$.

Dyslipidemia is observed in patients with DM and microalbuminuria. Total cholesterol increases significantly throughout with albuminuria. ${ }^{8}$ One researcher found hypercholesterolemia $(60 \%) .{ }^{9}$ in type II diabetic patient with albuminuria But one study reported that there was statistically insignificant correlation between microalbuminuria and total serum cholesterol in type II diabetic patients and it was present in $18.5 \%$ patients. ${ }^{10}$

Among the complications, cardiovascular mortality and morbidity carries a significant weightage and is the leading and most commonly encountered complication in diabetic lot. The increased risk of cardiovascular disease is associated with lipid abnormalities with the most common pattern consisting of elevated level of total cholesterol. Microalbuminuria, an early marker of diabetic nephropathy has been found to be associated with hypercholesterolemia in different studies. Microalbuminuria can be used as an early predictor of dyslipidemia. The rationale of this study is to find the correlation between microalbuminuria in newly diagnose diabetic and dyslipidemia in these patients so that patients can be managed at early stages and can prevented from severe cardio vascular morbidities and mortality. There are no local study available on this correlation and international studies showing variable results.

\section{Materials and Methods}

Sample size of 200 cases was calculated with $95 \%$ confidence level, $6 \%$ level of significance and taking expected percentage of microalbuminuria i.e. $24.2 \%{ }^{11}$ in newly diagnosed type-2 diabetic patients with nonprobability purposive sampling. Patients having age range $40-80$ years of either gender and with diagnosed diabetes mellitus (BSR > $200 \mathrm{mg} / \mathrm{dl}$ ) 2 hours post prandial not more than 3 months were included in the study. Patients having Overt proteinuria revealed by dipstick, patients with elevated serum creatinine before diagnosis of diabetes, patients with medical record of cardiac problem (assessed through ECG), patients with history of smoking or alcohol drinking and patients with haematuria (> 2 RBC) or UTI (pus cells > 4) were excluded. These 200 patients were enrolled in the study. Informed consent was obtained. Demographic information (Name, age, sex, address and contact) of each patient was noted. Urine was obtained and samples were sent to the laboratory of the hospital and reports were assessed. If the value of albumin is raised in urine analysis (as per operational definition) then albuminuria was labeled. In patients who were diagnosed as with albuminuria, total cholesterol was noted. If cholesterol was > $240 \mathrm{mg} / \mathrm{dl}$, then hypercholesterolemia was labeled by researcher himself. The collected data was analysed statistically by using SPSS version 20. Quantitative variables like age was presented in form of mean \pm S.D. Qualitative variables like gender and albuminuria was presented in form of frequency and percentage. Frequency and percentage were also calculated for hypercholesterolemiain diabetic patients with albuminuria. Data was stratified for age and gender.

\section{Results}

200 patients were recruited to determine the frequency of microalbuminuria and dyslipidemia in newly diagnosed diabetic patients. Age distribution of the patients was calculated and presented in Table 1, where most of the patients were recorded between $40-50$ years of age i.e. $38 \%(n=76), 27.5 \%(n=55)$ were recorded between $51-60$ years, $21 \%(n=42)$ were between $61-70$ years and $13.5 \%(\mathrm{n}=27)$ were recorded between $71-80$ years of age, the age range was 40-80 years, Mean \pm SD was calculated as $54.87 \pm$ 4.23 years. Frequency of microalbuminuria in newly diagnosed diabetic patients was recorded in $9.5 \%$ $(\mathrm{n}=76)$ while $62 \%(\mathrm{n}=124)$ were not presented with microalbuminuria (Table 2). Frequency of dyslipidemia in newly diagnosed diabetic patients out of 19 cases was recorded in $63.16 \%(\mathrm{n}=12)$ while $36.84 \%$ $(\mathrm{n}=7)$ had no findings of dyslipidemia (Table 3$)$. Stratification for frequency of dyslipidemia in newly diagnosed diabetic patients with microalbuminuria with regards to age was recorded, out of 12 cases, $8.33 \%(\mathrm{n}=1)$ were between $40-50$ years, $25 \%(\mathrm{n}=$ 3) between $51-60$ years, $41.67 \%(n=5)$ between $61-70$ years and $25 \%(n=3)$ between $71-80$ years of age (Table 4). Stratification for frequency of dys- 
lipidemia in newly diagnosed diabetic patients with microalbuminuria with regards to gender was recorded where out of 12 cases, $58.33 \%(\mathrm{n}=7)$ were male while $41.67 \%(\mathrm{n}=5)$ were females (Table 5).

Table 1: Distribution of Dyslipidemia in Patients According to Age.

\begin{tabular}{|c|c||c|c||}
\hline $\begin{array}{c}\text { Age(in } \\
\text { Years) }\end{array}$ & $\begin{array}{c}\text { No. of } \\
\text { Patients }\end{array}$ & $\begin{array}{c}\text { No. of Patients } \\
\text { Dyslipidemia }\end{array}$ & $\%$ \\
\hline \hline $40-50$ & 76 & 1 & 8.33 \\
\hline $51-60$ & 55 & 3 & 25 \\
\hline \hline $61-70$ & 42 & 5 & 41.67 \\
\hline \hline $71-80$ & 27 & 3 & 25 \\
\hline \hline Total & 200 & 12 & 100 \\
\hline
\end{tabular}

Table 2: Frequency of Microalbuminuria in Newly Diagnosed Diabetic Patients and Dyslepidemia $(n=200)$.

\begin{tabular}{|c||c|c||c|c||}
\hline $\begin{array}{c}\text { No. of } \\
\text { patients }\end{array}$ & $\begin{array}{c}\text { Microal- } \\
\text { buminuria }\end{array}$ & $\%$ & $\begin{array}{c}\text { Dyslipidemia in } \\
\text { Microalbuminuria }\end{array}$ & \% \\
\hline \hline Yes & 19 & 9.5 & 12 & 63.16 \\
\hline \hline No & 181 & 90.5 & 7 & 36.84 \\
\hline Total & 200 & 100 & 19 & 100 \\
\hline
\end{tabular}

\section{Discussion}

Prevalence of microalbuminuria has been studied worldwide with variable results. ${ }^{12}$ Earlier studies on Asia immigrant Indians and native Indians have suggested a high prevalence of microalbuminuria. Gupta et al reported a prevalence of $26.6 \%,{ }^{13}$ while John et al reported a prevalence of $19.7 \% .^{12}$ In contrast the results from white UK population revealed a prevalence of microalbuminuria of $7 \%-9 \% .^{13}$

We planned this study to determine correlation between microalbuminuria in newly diagnose diabetic and dyslipidemia in these patients so that patients can be managed at early stages and can prevented from severe cardio vascular morbidities and mortality as we recorded no local study available on this correlation and international studies showing variable results.

In our study, the common age was calculated as 54.87 \pm 4.23 years, $57.5 \%(\mathrm{n}=117)$ were male and $42.5 \%$ $(\mathrm{n}=83)$ were females, frequency of microalbuminuria in newly diagnosed diabetic patients was recorded in 9.5\% ( $\mathrm{n}=19)$ while $90.5 \%(\mathrm{n}=181)$ were not presented with microalbuminuria, while frequency of dyslipidemia in newly diagnosed diabetic patients out of 19 cases was recorded in $63.16 \%(\mathrm{n}=12)$ while $36.84 \%(\mathrm{n}=7)$ had no findings of dyslipidemia.

The findings of the study are in agreement with a study which reveals $7 \%$ having microalbuminuria at the time of diagnosis. ${ }^{5}$ In another study, the prevalence of microalbuminuria in diabetic patients was $24.2 \%{ }^{6}$ which is inconsistent with our study, the reason behind this inconsistency is unknown. Another study, among diabetic patients hypercholesterolemia was more frequent in patients compared with diabetic patients without microalbuminuria $(62.1 \% \text { vs. } 36.5 \%)^{7}$. These findings are in agreement with the findings of the current study.

Shonima Venugopal and colleagues ${ }^{14}$ who recorded Risk Factor Analysis and Prevalence of Microalbuminuria among Type 2 Diabetes Mellitus Subjects and recorded high prevalence of dyslipidemia among the subjects.

Tseng $\mathrm{CH}^{15}$ evaluated the lipid abnormalities associated with different stages of albuminuria in type 2 diabetic patients and recorded that a differential dyslipidemia is observed for microalbuminuria and macroalbuminuria. Apo (B) and $L p(a)$ increase at the stages of microalbuminuria and macroalbuminuria, respectively. However, TG increases significantly throughout the three stages of albuminuria.

An intriguing observation was made by Wendy $\mathrm{P}$ and colleagues. ${ }^{16}$ They documented that in type 2 diabetics, dyslipidemia is a substantial problem which in turn leads to cardiovascular complications. There is a spectrum of lipid abnormalities that are present in diabetics, amongst which hypertriglyceridemia and low levels of high-density lipoprotein cholesterol are the leading abnormalities. On the contrary, Low-density lipoprotein levels in these patients are often similar to control population, although there is a possibility that qualitative difference might exist in diabetics that make them a high risk for development of cardiovascular disease. However, the results of the study are primary in our local population which needs some other trials to confirm these findings so that patients can be managed at early stages and can prevented from severe cardio vascular morbidities and mortality. 


\section{Conclusion}

The results of the study concluded that frequency of microalbuminuria among patients of newly diagnosed type-2 diabetic mellitus is in agreement with other studies while the frequency of dyslipidemia in diabetic-II patients with microalbuminuria was also recorded higher which needs special attention of physicians for its timely management so that patients can be prevented from severe cardio vascular morbidities and mortality.

\section{References}

1. Diabetes facts. The prevalence of diabetes has reached epidemic proportions. [online] 2010 [cited2012]. Available at: http://www.worlddiabetesfoundation.org/composite35.htm.

2. Melmed S, PolonskyKS, Larsen PR, Kronenberg HM. Williams textbook of endocrinology. 12th ed. Philadelphia: Elsevier/Saunders, 2011: 1371-435.

3. International Diabetes Federation. Diabetes Atlas. 3rd ed. Brussels, Belgium: International Diabetes Federation: 2006.

4. Aziz S, Noorulain W, Zaidi U R, Hossain K, Siddiqui I A. Prevalence of overweight and obesity among children and adolescents of affluent schools in Karachi. J Pak Med Assoc. 2009; 59: 35-8.

5. Ahmedani MY, Hydrie MZI, Iqbal A, Gul A, Mirza WB. Prevalence of Microalbuminuria in Type 2 Diabetic Patients in Karachi, Pakistan: a Multi-center Study. J Pak Med Assoc. 2005; 55 (9): 382-6.

6. Ahmedani MY, Fawwad A, Basit A, Hydrie ZI. Microalbuminuria prevalence study in hypertensive patients with type 2 diabetes in Pakistan. J Ayub Med Coll Abbottabad, 2008; 20 (3): 117-20.
7. Wang SH, Wang L, Zhou Y, Guo YJ, Yuan Y, LiFF, et al. Prevalence and control of dyslipidemia among diabetic patients with microalbuminuria in a Chinese hospital. Diabetes and Vascular Disease Research 2012.

8. Tseng $\mathrm{CH}$. Differential dyslipidemia associated with albuminuria in type 2 diabetic patients in Taiwan. Clin Biochem. 2009; 42 (10-11): 1019-24.

9. Tien KJ, Tu ST, Chen HC, Hsiao JY, Hsieh MC. Triglycerides are independently associated with albuminuria in Taiwanese type 2 diabetic patients. J Endocrinol Invest. 2011. [Epub ahead of print]

10. Afkhami-Ardekani M, Modarresi M, Amirchaghmaghi E. Prevalence of microalbuminuria and its risk factors in type 2 diabetic patients. Indian J Nephrol. 2008; 18 (3): 112-7.

11. Ko GT, Chan JC, Lau M, Cockram CS. Diabetic microangiopathic complications in young Chinese diabetic patients: A clinic based cross sectional study. J Diabetes Compl. 1999; 13: 300-6.

12. Allawi J, Rao PV, Gilbert R. Microalbuminuria in noninsulin - dependent diabetes: Its prevalence in Indian compared with Europid patients. Br Med J. 1988; 296: 462-4.

13. Gupta DK, Verma LK, Dash SC. Prevalence of microalbuminuria in diabetes: a study from north India. Diabetes Res Clin Pract. 1991; 12: 125-8.

14. Venugopal S, Uma M Iyer. Risk Factor Analysis and Prevalence of Microalbuminuria among Type 2 Diabetes Mellitus Subjects: The Need for Screening and Monitoring Microalbumin. Asian J ExpBiol Sci. 2010; 1 (3): 652-9.

15. Tseng $\mathrm{CH}$. Differential dyslipidemia associated with albuminuria in type 2 diabetic patients in Taiwan. Clin Biochem. 2009; 42 (10-11): 1019-24.

16. Wendy P. William F. Dyslipidemia in Patients with Type 2 Diabetes. Relationships between Lipids, Kidney Disease and Cardiovascular Disease. Clinical Chemistry and Laboratory Medicine, 2005; 41: 1174-81. 